\title{
Gerenciamento de dados marinhos no contexto brasileiro
}

\author{
Luis Américo Conti ${ }^{1,4}$, Mariana Cabral de Oliveira ${ }^{2,4}$, \\ Tiago Egger Moellwald Duque Estrada ${ }^{3}$ \& Antônio Carlos Marques ${ }^{2,4}$ \\ ${ }^{1}$ Escola de Artes, Ciências e Humanidades - EACH, Universidade de São Paulo - USP, \\ Av. Arlindo Bettio, 1000, CEP 03828-000, São Paulo, SP, Brasil \\ ${ }^{2}$ Instituto de Biociências - IB, Universidade de São Paulo - USP, Rua do Matão, \\ Travessa 14, 321, CEP 05508-900, São Paulo, SP, Brasil \\ ${ }^{3}$ Programa Biota/FAPESP, Departamento de Biologia Vegetal, Instituto de Biologia, \\ Universidade Estadual de Campinas - UNICAMP, CP 6109, CEP 13083-970, Campinas, SP, Brasil \\ ${ }^{4}$ Núcleo de Pesquisa em Biodiversidade Marinha - NP-BioMar, Centro de Biologia Marinha - CEBIMAR, \\ Universidade de São Paulo - USP, Rod. Manuel Hipólito do Rego, Km 131,5, \\ CEP 11600-000, São Sebastião, SP, Brasil \\ ${ }^{4}$ Autor para correspondência: Luis Américo Conti,e-mail: lconti@usp.br
}

CONTI, L.A., OLIVEIRA, M.C., ESTRADA, T.E.M.D. \& MARQUES, A.C. Marine data management in the Brazilian context. Biota Neotrop. 13(2): http://www.biotaneotropica.org.br/v13n2/en/abstract?pointofvie $\mathrm{w}+\mathrm{bn} 00113022013$

Abstract: In a context of rapidly growing demand of information and production of technical and scientific
knowledge about biotic and abiotic parameters of marine and coastal environments, the process of design
integrated databases programs and geo-portals for disseminating information becomes imperative. Although
isolated efforts have been proposed in recent years in Brazil, the creation of broadly accessible databases seems
inevitable. Furthermore, the peculiarities of acquisition and processing marine data (expensive, complex and
dynamic) demand an optimization of time and resources for new research projects and knowledge basis. In
this work we address this issue contextualizing the demands for the implementation and marine data modelling
projects, focusing on theoretical and practical aspects providing guidelines and connectivities establishing future
efforts towards the dissemination of marine and coastal information in Brazil. Keywords: marine and coastal environments, database, geoportals, habitat mapping.

CONTI, L.A., OLIVEIRA, M.C., ESTRADA, T.E.M.D. \& MARQUES, A.C. Gerenciamento de dados marinhos no contexto brasileiro. Biota Neotrop. 13(2): http://www.biotaneotropica.org.br/v13n2/pt/abstract?pointofvie $\mathrm{w}+\mathrm{bn} 00113022013$

Resumo: Em um contexto de franco crescimento de demanda e produção de conhecimento técnico e cientifico a respeito de parâmetros bióticos e abióticos de ambientes marinhos e costeiros, a concepção de programas de desenvolvimento de bancos de dados e geo-portais de disseminação de informação torna-se imperativa. Embora apenas esforços pontuais tenham sido propostos nos últimos anos, a criação de bases de dados de acesso mais amplo parece inevitável tendo em vista as peculiaridades práticas de aquisição e processamento de dados marinhos: caros, complexos e dinâmicos. Este trabalho pretende abordar tal questão contextualizando as necessidades e demandas para projetos desta natureza, focando aspectos teóricos e práticos da implementação de modelos de dados marinhos propondo diretrizes para que esforços futuros sejam estabelecidos visando a disseminação da informação marinha e costeira no Brasil.

Palavras-chave: ambiente marinho e costeiro, banco de dados, geoportais, mapeamento de habitats. 


\section{Introdução}

O mapeamento e a caracterização sistemática de aspectos bióticos e abióticos das regiões marinhas e costeiras tiveram grande desenvolvimento desde a segunda metade do século XX, acompanhados do crescimento populacional de regiões costeiras e da demanda por recursos naturais marinhos. Avanços na tecnologia da informação aliados ao desenvolvimento de sistemas compartilhados de armazenamento de dados permitem um acesso sem precedentes à informação biogeográfica marinha, em resoluções de uma a três ordens de magnitude maior do que era possível anos atrás (Soberón $\&$ Peterson 2004). No entanto, a criação de bases de dados para o estudo da biodiversidade marinha não deve se limitar, simplesmente, à criação de bancos de dados ou inventários, mas, principalmente, buscar abranger o desenvolvimento de métodos e abordagens que permitam a integração de uma grande quantidade de informações de natureza, escala, precisão e estrutura distintas, e que sejam suscetíveis a articulação e interoperabilidade espacial e metodológica.

Neste contexto, o objetivo deste trabalho é apresentar contribuições para o desenvolvimento de uma base teórica que sustente a implementação de projetos voltados à sistematização de dados marinhos, discutindo os aspectos principais de organização e distribuição de dados, incluindo a experiência de programas internacionais. O desenvolvimento deste estudo foi fruto da discussão entre pesquisadores e gerenciadores de bancos de dados marinhos e costeiros em evento realizado na Fundação de Amparo à Pesquisa do Estado de São Paulo (FAPESP), em abril de 2012, no contexto do Programa Biota (Marine Data Management - http://www. fapesp.br/6873). Naquela oportunidade foram abordadas diversas questões a respeito da situação atual no Brasil frente aos desafios de desenvolvimento de projetos de sistematização e integração de dados oceanográficos e apontadas algumas das questões chaves que ainda dificultam a adoção e implementação de grandes programas de gerenciamento de dados a saber:

- Compartilhamento de dados: vários projetos de pesquisa e de mapeamento de dados marinhos da plataforma continental brasileira foram desenvolvidos nas últimas décadas, gerando uma grande quantidade de informação. Uma parte desta informação foi publicada em trabalhos científicos, porém apenas uma quantidade pequena da mesma foi incluída em bases de dados que servem como plataformas para a disseminação do conhecimento. Estas plataformas são a base para meta-análises que busquem padrões inexplorados, inclusive os relacionados à sinergia entre os dados de diferentes naturezas. Este cenário é parcialmente explicado pela ausência de esforços coordenados entre universidades/centros de pesquisas, órgãos governamentais e não-governamentais que tenham por objetivo, ou práxis, a estruturação e normatização de dados gerados nestas pesquisas. Assim, a realidade atual mostra uma pulverização de informações desconectadas, uma perda substancial de dados primários e a impossibilidade de manipulação e acesso às informações. O prejuízo científico, logístico e financeiro desta realidade é imenso. O cenário brasileiro, nesse sentido, é antagônico as tendências internacionais, que têm incentivado programas de organização e disseminação de informações como base para pesquisas científicas, principalmente no que se refere à disseminação de dados de caráter ambiental. $\mathrm{O}$ desenvolvimento destes programas não está circunscrito a inventários, mas relaciona-se à criação de uma nova "mentalidade" no que se refere à necessidade de compartilhamento de dados, sejam eles brutos ou pós processados.

- Infraestrutura de Dados Espaciais - IDE: Um aspecto essencial de ações de articulação e interoperabilidade de dados se refere à necessidade de criação de normas e padrões semânticos que estabeleçam uma base comum aos diferentes projetos, além de reunir aspectos de diversas disciplinas e ramos do conhecimento sob um mesmo guarda-chuva conceitual. Uma IDE é composta por políticas, tecnologias e padrões que interconectam usuários de informação espacial. Portanto, a implementação de um programa de IDE deve visar a organização da informação geográfica, implicando no desenvolvimento de padrões centralizados de gerenciamento e distribuição hierarquizados, em um âmbito local, regional, nacional e supranacional (Phillips \& Williamson 1999). Exemplos internacionais de sucesso na implementação de IDEs são notórios, em especial para dados marinhos. Destacam-se, nesse contexto, o INSPIRE (Infrastructure for Spatial Information in the European Comunity; http://inspire.jrc.ec.europa.eu), que serviu de base para o programa especifico de dados marinhos europeu EMODNet (European Marine Observation and Data Network; http://bio. emodnet.eu/) (detalhes em Proctor \& Howarth 2008, Meiner, 2010). No Brasil, esforços, em um nível nacional, têm sido organizados a partir do processo de implementação do programa INDE/Brasil (Infraestrutura Nacional de Dados Espaciais; http://www.inde.gov.br/), coordenado pela Comissão Nacional de Cartografia (CONCAR), cujo objetivo é a ordenação e gestão de armazenamento e o acesso dos dados e metadados geoespaciais em nível nacional (Brasil, 2008). Entretanto, no âmbito do INDE, não existem diretrizes específicas no que se refere a dados marinhos ou costeiros. É importante salientar que o desenvolvimento de uma IDE não deve se restringir aos aspectos técnicos e operacionais relativos às tecnologias de gerenciamento de dados, mas também a ações políticas e administrativas que propiciem uma ampla abrangência e disseminação de sua essência e documentação (metadados).

- Programas de Interoperabilidade e Articulação - Além da normatização e estabelecimento de sistemas de gerenciamento e disseminação de dados (IDEs), a implementação de programas de integração de dados marinhos deve ser acompanhada de redes de projetos locais e de organização e cooperação de grupos de trabalho, especialmente em um país com as dimensões do Brasil. O ICAN (Internacional Coastal Atlas Network; http://ican.science.oregonstate.edu/) é um exemplo de rede de compartilhamento de experiências e normas em uma abordagem multidisciplinar, cujo principal objetivo é criar um meio de compartilhar dados e informações marinhas e costeiras via $W E B$. Outros exemplos de discussão e normatização de dados costeiros são Marine Metadata Interoperatibility Project (http://marinemetadata.org/), Network Marine Research Institutes and Documents - MARENET (http://www.marenet. de/MareNet/), NOAA National Marine Data Center (http:// www.lib.noaa.gov/index.html). Boa parte destes programas baseia-se na necessidade latente do desenvolvimento de pesquisas interdisciplinares e multi-temporais que sustentem o conhecimento sobre a dinâmica de ambientes marinhos e costeiros frente a um cenário de mudanças ambientais globais e, assim, auxiliem o desenvolvimento de estudos integrados visando ações de conservação e para políticas públicas. A UNESCO também tem desenvolvido um papel fundamental na organização de grupos de trabalho, por meio da Comissão Internacional de Oceanografia (IOC, da sigla em inglês). Dentre seus programas, o IODE (International Oceanographic Data and Information Exchange; http://www.iode.org/) subsidiando ações de gerenciamento e acesso a dados marinhos provendo suporte técnico, educacional e financeiro visando estabelecer bases sólidas para o desenvolvimento de um sistema de informação global de dados marinhos e costeiros em menos de uma década (Strain et al. 2006). 
As três questões (viz., compartilhamento, infraestrutura e interoperabilidade) devem ser abordadas de forma abrangente e objetiva para o desenvolvimento de políticas de disseminação de informações, uma vez que é imperativo que o conhecimento desenvolvido e acumulado ao longo de décadas de estudos marinhos torne-se acessível de maneira clara e eficiente para toda a sociedade. A viabilização de uma política de integração e disponibilização de dados permitirá que a comunidade científica que trabalha com o tema possa desenvolver hipóteses mais abrangentes e consolidadas a partir de programas e projetos de pesquisa multidisciplinares. A seguir são abordados dois aspectos conceituais discutidos no âmbito do Workshop sobre gerenciamento de dados marinhos que pretendem contribuir para a criação de tais programas: o "Planejamento e hierarquização de uma base de dados marinhos" e "A concepção de um programa de banco de dados marinhos".

\section{Planejamento e Hierarquização de uma Base de Dados Marinhos}

A organização de dados pode apresentar diversos níveis escalonados de análise referentes à sua integração (Figura 1).
Em um nível inferior de análise, os dados disponíveis são processados e interpretados de forma interdependente e pouco integrada tanto geograficamente (não articulados) quanto em termos de sua disciplinaridade e especificidade. Tal foco geralmente é sustentado por pesquisas temáticas que possuem pouco ou nenhum instrumento de gerenciamento e compartilhamento de informações. Fundamentalmente toda a pesquisa marinha e costeira no Brasil, até o início do século XXI, baseou-se em uma concepção de valorização de coleta de uma grande quantidade de dados, visando a caracterização de um aspecto específico de pesquisa (e.g. taxonomia, ecologia, geoquímica, geologia) e manutenção destes dados cativos para tais usos. Deste modo, um aspecto prioritário que permeia toda a discussão sobre novos projetos de gerenciamento de dados refere-se à necessidade de levantamento e catalogação de dados associados aos projetos já executados, uma vez que uma pequena parte destes encontra-se publicada, padronizada e disponível em publicações de acesso irrestrito.

Um segundo nível contempla a integração de dados dentro da mesma disciplina, ou em uma articulação de projetos contíguos baseados em coletas ou reunião de dados em um contexto de maior multidisciplinaridade, mesmo não havendo, necessariamente, análises integradas sensu stricto. Projetos de Atlas e trabalhos dirigidos como

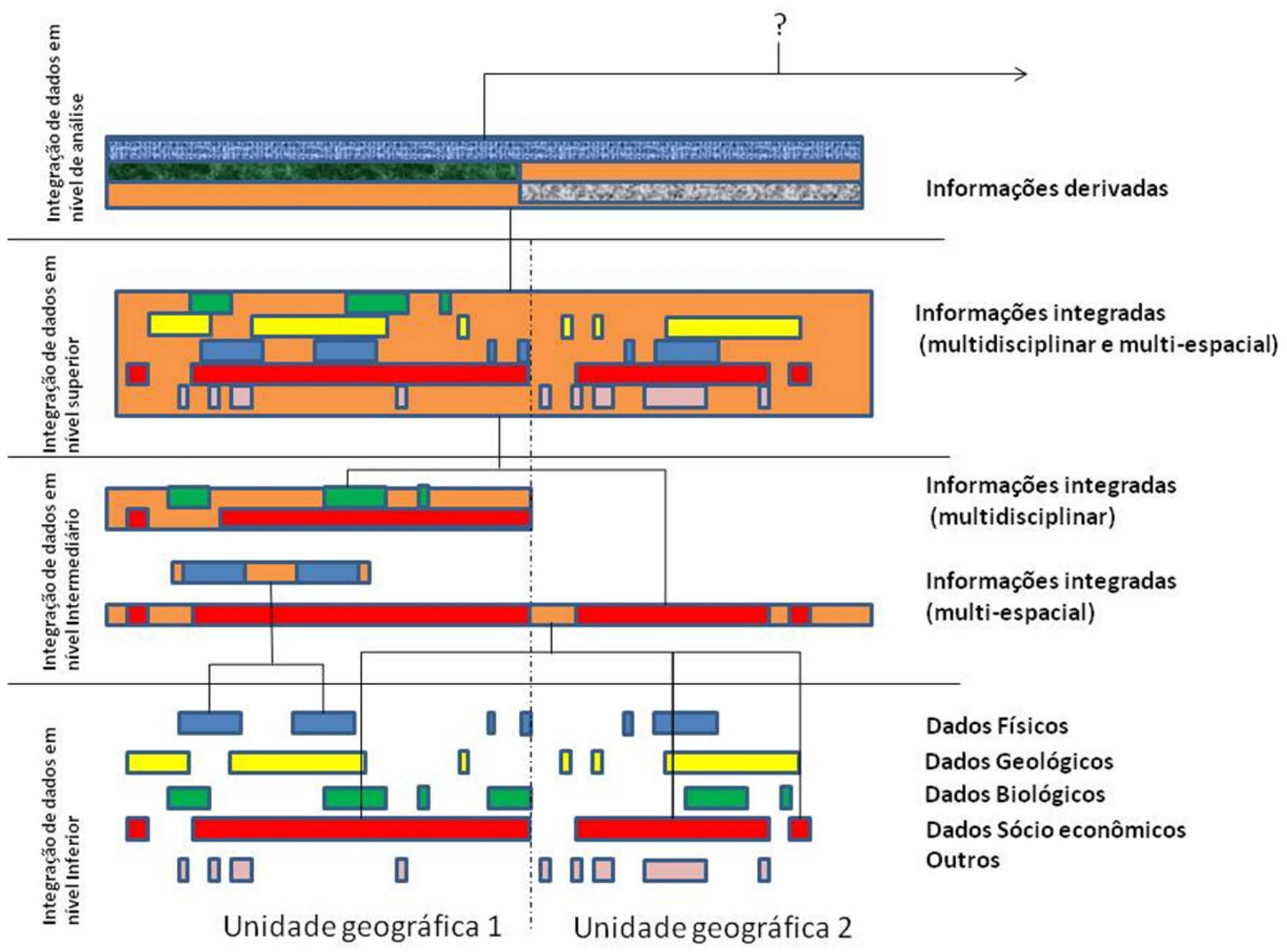

Figura 1. Estrutura conceitual de integração de dados marinhos e costeiros. Áreas geográficas estão dispostas no eixo X e sucessivos graus de interação dos dados no eixo Y. As cores representam diferentes áreas do conhecimento.

Figure 1. Conceptual Structure for marine and coastal data integration. Geographic areas is represented by the $\mathrm{X}$ axis where interaction degrees are represented by $\mathrm{Y}$ axis. Different colors indicate knowledge areas. 
o "Atlas Sedimentológico do Estado de São Paulo" (Rodrigues et al. 2001) ou do "Atlas Geográfico das Zonas Costeiras e Oceânicas do Brasil" (IBGE, 2011) englobam articulação de áreas contíguas em bases cartográficas comuns em diversos níveis de informações e, embora ofereçam, em algum nível, integração de dados de diferentes naturezas, normalmente são de uso e acesso restrito às suas instituições geradoras e mantenedoras, além de que, normalmente são divulgados somente os produtos finais (i.e. mapas, relatórios, artigos técnicos).

Um terceiro nível baseia-se em dados multidisciplinares e multiespaciais (articulados) em um estágio de análise superior, onde informações completamente distintas podem ser correlacionadas e disponibilizadas espacialmente - é o caso da maioria dos sistemas de inventários e bancos de dados espaciais baseados em Geoportais. Atualmente, a maior parte das discussões em torno dos aspectos técnicos e práticos de implementação de bancos de dados espaciais marinhos e costeiros encontra-se neste nível. Talvez os exemplos mais disseminados, em nível internacional de bancos de dados espaciais, sejam o OBIS (Ocean Biogeographic Information System; http:// www.iobis.org/) e o MGDA (Marine Geophysical Data Access; http://www.ngdc.noaa.gov/mgg/geodas). Em geral, tais bases são mantidas e geridas por universidades, centros de pesquisas ou agências governamentais, possibilitando acesso livre tanto aos dados brutos quanto às informações processadas. Iniciativas brasileiras com as mesmas características podem ser igualmente citadas, como o Banco Nacional de Dados Oceanográficos (BNDO), Banco de Dados Ambientais para a Indústria do Petróleo (Banpetro; http://www. bampetro.on.br), o SinBiota, Sistema de Informação Ambiental para o Programa Biota/FAPESP (http://sinbiota.biota.org.br) entre outras. A adoção destas bases pode ser uma solução eficiente para o resgate dos dados ora indisponíveis. Porém, a maior parte destes programas ainda não prevê a integração com bases de dados ambientais distintos, impossibilitando, por exemplo, uma correlação de dados geológicos, biológicos e hidrodinâmicos com o objetivo de planejamento e manejo de áreas marinhas protegidas, principalmente em escalas locais e regionais.

Em um nível mais alto, são incluídos os programas totalmente integrados, em que não há apenas uma normatização e organização de dados, mas também o desenvolvimento efetivo de ferramentas de análises de geoprocessamento combinadas que produzem, necessariamente, informações novas derivadas dos dados originais. Um exemplo de projetos deste caráter em áreas marinhas é o MESH (Mapping European Seabed Habitats - http://www.searchmesh. net), que teve por objetivo a determinação e classificação de habitats bentônicos em todas as regiões da plataforma continental européia. Seus produtos vão além do mapeamento e disponibilização de dados da biodiversidade porque visam a produção de novas informações ambientais baseadas na relação (muitas vezes não-lineares e diretamente correlacionáveis) entre padrões de níveis inferiores.

O pleno uso dos dados é alcançado apenas no nível mais elevado, uma vez que informações de diversas fontes e escalas de análises podem ser integradas e processadas, visando à geração de modelos preditivos complexos e altamente estruturados. Poucos exemplos mundiais têm tal nível de integração, mas estas bem sucedidas exceções têm se mostrado promissoras no que se refere a uma tentativa de compreensão do caráter extremamente diversificado do ambiente marinho e da interconexão dos processos atuantes. Estes níveis são invariavelmente dependentes de ações colaborativas de entrada de dados e necessitam de uma gerência ativa, não apenas para organizar e normatizar as informações (e necessariamente, precedida de uma base de IDEs) mas, também, para conceber e implementar novas ferramentas de análise. As organizações governamentais e não governamentais que lidam com gerenciamento de dados ou informações têm apostado no desenvolvimento de diversas ferramentas de compartilhamento no nível de análise para o futuro.

No Brasil, em oposição, a ausência de trabalhos com alto índice de integração para áreas marinhas e costeiras pode ser explicada por três fatores principais: (a) para ambientes marinhos, especialmente em áreas de bacias oceânicas profundas, ainda há ausência de informações básicas como batimetria de detalhe ou cobertura sedimentar, que serviriam de base e suporte para análises mais complexas, como de habitats ou de metapopulações. Além disso, boa parte das informações coletadas tem caráter pouco "espacializável”, isto é, são descrições taxonômicas derivadas de coletas pontuais sem informações geográficas precisas. (b) A ausência de esforços governamentais de integração e gerenciamento de informações, principalmente em função da falta de base institucional para a concepção e execução de projetos de desenvolvimento de sistemas de coleta, organização e gestão de dados e metadados. (c) A falta de uma política clara relacionada à implementação e aplicação de Infraestrutura de Dados Espaciais (IDE) no que se refere a dados ambientais marinhos no Brasil, base para ações de interoperabilidade como padronização de semântica e ontologias.

\section{A Concepção de um Programa de Banco de Dados Marinhos}

Dentro do contexto discutido, a concepção de um programa de alto nível de integração de dados marinhos em um banco de dados compartilhado e georreferenciado em ambiente Web deve passar pela criação de, ao menos, três estágios organizados e interconectados de modo a garantir a interoperabilidade e acesso as informações disponibilizadas (Figura 2).

Estágio 1: Organização de dados e construção de um catálogo de metadados.- Esta fase talvez seja a mais trabalhosa em termos de tempo e esforços, uma vez que a necessidade de organização de uma grande quantidade de dados e informações em posse de pesquisadores, entidades públicas e privadas, demanda esforços conjuntos e coordenados. Algumas das dificuldades inerentes à fase de organização de dados são associadas à determinação de parâmetros de metadados, ou de qualidade dos dados (data quality), tais como fatores de precisão de posicionamento, descrição de métodos de coleta, de armazenamento, de tratamento em laboratório, e a integridade dos dados. Tais informações, quando existentes, além de catalogadas, devem também ser estruturadas segundo padrões internacionais de metadados por meio de um conjunto de informações essenciais para ajudar na localização, qualificação e entendimento dos dados geoespaciais, ou seja, da implementação de um projeto de infraestrutura de dados espaciais (IDE). Entre as organizações internacionais que se ocupam de trabalhar com padronização de dados referentes ao espaço geográfico, o Open Geospatial Consortium

- OGC e a Global Spatial Data Infrastructure Association - GSDI têm papéis destacados (Hubner \& Oliveira, 2008). Diversos trabalhos têm sido publicados explorando detalhes a respeito da base para o desenvolvimento de IDE (detalhes em Nebert 2001, Granell et al. 2007, Janowicz et al. 2010, e especificamente para dados marinhos e costeiros Strain 2004, Bartlett et al. 2004, Wright 2009, Lavoi et al. 2010, Kulawiak et al. 2010 entre outros).

A estruturação de um catálogo de metadados tem como consequência direta, a identificação de vazios de conhecimento, tanto em termos espaciais quanto em termos conceituais, de modo que, mesmo sem a intenção imediata de construção de um banco de dados, a organização de catálogos de metadados poderia ser utilizada para nortear a temática da pesquisa acadêmica visando o direcionamento de editais de fomento e evitando a coleta de informações redundantes.

Estágio 2: Implementação de bancos de dados e de estratégias de compartilhamento.- $\mathrm{O}$ desenvolvimento de bancos de dados 
Estágio 1

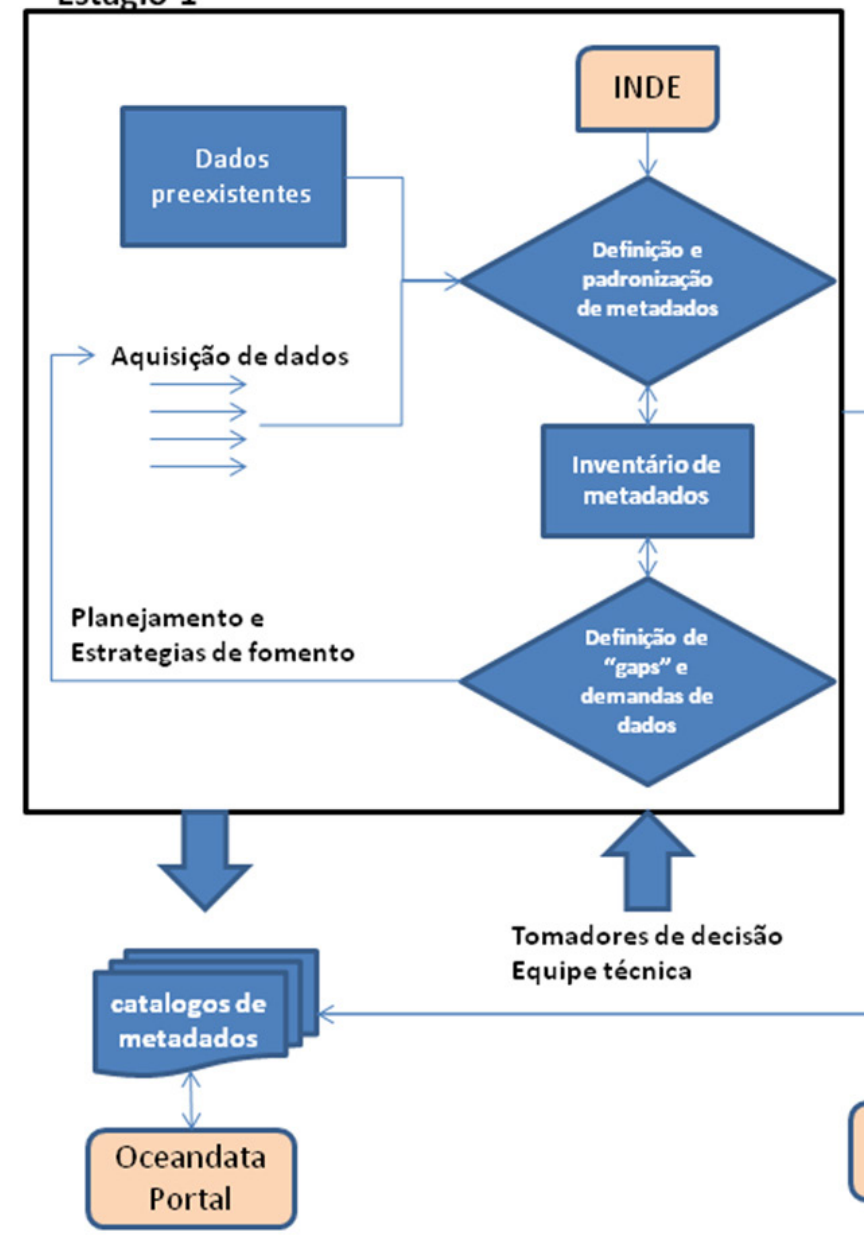

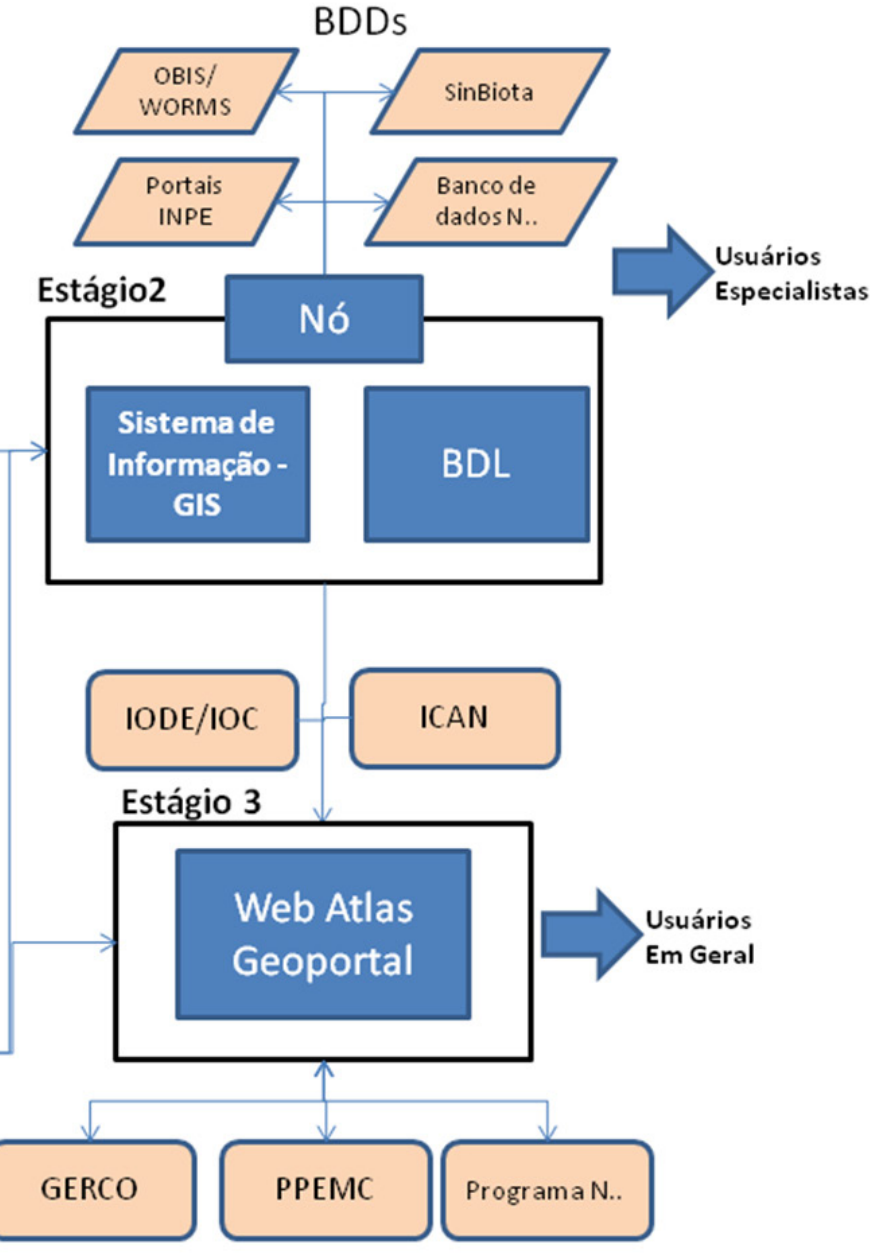

Figura 2. Modelo de um fluxograma para a implementação de um projeto de pesquisa visando a implementação de um programa de integração de dados marinhos para o estado de São Paulo.

Figure 2. An organizational framework for the implementation a research project for Marine and Coastal data integration model at São Paulo State.

georreferenciados distribuídos em rede pode seguir duas formas básicas de estrutura: o que se chama de "Bancos de Dados Localizados" (BDL), em que o conjunto de informação, dados e metadados fica estabelecido fisicamente em um centro (servidor) e os "Bancos de Dados Distribuidores" (BDD), em que a informação é pulverizada em diversos BDLs e agregada em um nó que a codifica e integra. Boa parte dos sistemas concebidos nos últimos anos tem um caráter híbrido, com parte da informação localizada "in house" em servidores e, simultaneamente, agregando informações como um nó. Independente do caráter local ou distribuído, os sistemas de banco de dados georreferenciados compartilhados devem possuir, necessariamente, um sistema de semântica e ontologia de metadados que permita o usuário acessar, manipular e analisar informações geográficas dentro de normas e padrões estabelecidos. Por exemplo, ao buscar um termo "linha de costa" há a possibilidade de retorno de várias informações com níveis de precisão e formatos, como arquivos vetoriais derivados de sistema GEBCO (General Bathymetric Charts of The Oceans), linhas digitalizadas a partir de cartas topográficas, linhas de referência (como a da marinha ou do IBGE). Ademais, o termo "linha de costa" deve estar organizado de modo a ser relacionado com referências como "linha costeira", "litoral", assim como os termos em inglês "shoreline" e "coastline".

Ao se estabelecer um banco de dados tipo BDD ou Híbrido, também é necessário que haja um padrão claro de interoperabilidade com sistemas BDL alimentadores. No caso de um sistema envolvendo a biodiversidade marinha, é imperativo que haja uma coordenação com sistemas internacionais, como o WoRMS (World Register of Marine Species) e o OBIS, e nacionais, como o SinBiota.

Estágio 3: Desenvolvimento de um geoportal de divulgação e distribuição da informação.- A partir do estabelecimento de uma infraestrutura de dados e metadados, envolvendo tecnologias, políticas e normas estabelecidas, a distribuição da informação georreferenciada via Internet, a partir de um Web-Atlas, tem sido a base para soluções de compartilhamento e disseminação de informações.

Um portal de Informações Geográficas, ou Web-Atlas, tem como objetivo central facilitar o acesso aos dados espaciais e disseminar o uso da informação, principalmente no que se refere a aspectos como a busca de informações a partir de uma determinada localização na Terra, determinar padrões e critérios de distribuição de variáveis, prover serviços de mapeamento interativo, estabelecimento de demandas e de lacunas nas informações. Exemplos de projetos de grande porte foram desenvolvidos, principalmente, na Europa e nos Estados Unidos, o que também aumentou consideravelmente a acessibilidade de dados espaciais marinhos e costeiros por nãoespecialistas. Combinado com as necessidades cada vez mais urgentes da população crescente que ocupa as regiões costeiras, isso resultou no crescimento de um grupo de nicho de Web-Atlas marinhos e costeiros ao redor do mundo. O Livro Verde de 2006 sobre a política marítima 
futura da União Européia, por exemplo, refere-se aos atlas costeiros e marinhos como um instrumento fundamental de ordenamento de territórios nacionais, ilustrando o crescente reconhecimento internacional do potencial utilidade da sistematização de dados marinhos (O'Dea et al. 2011).

Uma série de projetos de atlas costeiros e marinhos tem se desenvolvido dentro deste contexto, além de ações supranacionais, com amplo apoio da agências ambientais nacionais e locais, envolvendo a integração de dados bióticos e abióticos como base para o estabelecimento de políticas ambientais comuns. Tais esforços focam, principalmente, no desenvolvimento de métodos e abordagens que permitam a integração de uma grande quantidade de informações de natureza, escala, precisão e estrutura distintas. Exemplos como os projetos MIDA (Marine Irish Coastal Atlas - http://mida.ucc.ie/ contents.htm), Belgian Coastal Atlas (De Kustatlas - http://www. kustatlas.be), Venice Coastal Atlas (http://atlante.silvenezia.it/), Oregon Coastal Atlas (http://www.coastalatlas.net) são cada vez mais frequentes e, em muitos casos, servem de suporte ao desenvolvimento de programas mais amplos e diversificados, como o OCEANGIS da NOAA (EUA; http://www.pmel.noaa.gov/vrl/OceanGIS).

A criação de um Web-Atlas (e da infraestrutura e da organização de bancos de dados que o precede) permite não apenas a entrega (delivery) de dados e informações, mas também a realização de análises e processamento. Embora boa parte dos sistemas atuais ainda apresente um caráter embrionário a este respeito, o consenso dentro da comunidade científica é que a disponibilização de uma quantidade cada vez maior de dados aliado ao desenvolvimento de processos de computação em rede (cloud computing) desloque a maior parte das análises e processos de manipulação de dados para a própria rede e, em consequência, os sistemas de Web-Atlas podem servir como base para projetos de pesquisa derivados que visem a análise e aplicação de dados, principalmente no que se refere ao gerenciamento costeiro e programas de conservação, tal como o Plano Nacional de Gerenciamento Costeiro (GERCO) ou Programas de Planejamento Espacial Marinho e Costeiro (PPEMC).

\section{Conclusões}

Há uma necessidade evidente de definir uma estratégia para a participação efetiva do Brasil no esforço internacional de conhecer melhor e contribuir efetivamente para a descrição, conservação, gerenciamento e aproveitamento racional e sustentado do ambiente marinho. O desenvolvimento de tecnologias de gerenciamento de dados espaciais é um aspecto fundamental para que a comunidade científica brasileira possa ter uma atuação mais ativa e diretamente relevante para o conhecimento da biodiversidade marinha.

Torna-se necessário, desta maneira, uma ampla adequação e estruturação das redes de informações e de dados para que sejam compatíveis com tendências da pesquisa científica global. Grandes programas de pesquisa, como o estudo de genoma de organismos marinhos (e.g., GIGA) e a investigação e monitoramento multidisciplinar de mudanças climáticas necessitam de bancos de dados cada vez mais robustos e sistemas de compartilhamento de informação hierarquizados e abertos em diversos níveis de alimentação e uso. É necessário, em termos estratégicos, que sejam propostas diretrizes que promovam políticas integradas de coleta e organização de dados marinhos em uma ação 'top-down', e tal demanda deve estar em consonância com uma expectativa da comunidade científica ou privada, o que geraria também uma ação 'bottom-up'. Somente a concatenação destes interesses pode garantir uma política sustentável de gerenciamento e acesso aos dados e informações das áreas costeiras e oceânicas do Brasil.

\section{Referências Bibliográficas}

BARTLETT, D., LONGHORN, R. \& GARRIGA, M. 2004. Marine and Coastal Data Infrastructures: a missing piece in the SDI puzzle. In 7th Global Spatial Data Infrastructure conference. Bangalore.

BRASIL. Presidência da República. 2008. Decreto n ${ }^{\circ}$ 6.666, de 27 de novembro de 2008. Institui a Infraestrutura Nacional de Dados Espaciais - INDE. Diário Oficial da República Federativa do Brasil, Braslília, nov.

GRANELL, C., GOULD, M., MANSO, M.A. \& BERNABÉ, M.A. 2007. Spatial Data Infrastructures. In Handbook of Research on Geoinformatics (H. Karimi, ed.). Information Science Reference, Hershey. INSPIRE Portal. http://www.ec-gis.org/inspire/.

HUBNER, C.E. \& OLIVEIRA, F.H. 2008 Gestão da Geoinformação em Implementações Multiusuários. In Congresso Brasileiro de Cadastro Técnico Multifinalitário. UFSC, Florianópolis, 10p.

INSTITUTO BRASILEIRO DE GEOGRAFIA E ESTATÍSTICA - IBGE. 2011. Atlas geográfico das zonas costeiras e oceânicas do Brasil. IBGE, Rio de Janeiro.

JANOWICZ, K., SCHADE, S., BRÖRING, A., KESSLER, C., MAUE, P. \& STASCH, C. 2010. Semantic Enablement for Spatial Data Infrastructures. Trans. GIS 14:111-129. http://dx.doi.org/10.1111/j.14679671.2010.01186.x

KULAWIAK, M., CHYBICKI, A. \& MOSZYNSKI, M. 2010. Web-based GIS as a tool for supporting marine research. Mar. Geodesy 36(8):1069-1080

LAVOI, T., MURPHY, J., SATALOFF, G., LONGHORN, R., MEINER, A., UHEL, R., WRIGHT, D.J. \& DWYER, E. 2010. Coastal Atlases in the Context of Spatial Data Infrastructures. In Coastal Informatics: Web Atlas Design and Implementation (D.J. Wright, N. Dwyer \& V. Cummins, eds.). IGI Global, p.239-255. http://dx.doi.org/10.4018/9781-61520-815-9.ch016

MEINER, A. 2010. Integrated maritime policy for the European Union - consolidating coastal and maritime information to support maritime spatial palnning. J. Coastal Conserv. 14: p. 1-11. http://dx.doi. org/10.1007/s11852-009-0077-4

NEBERT, D.D. 2001. Developing Spatial Data Infrastructures: The SDI Cookbook. version 1.0 (GSDI-Technical Working Group). http://www. gsdi.org/pubs/cookbook/cookbook0515.pdf.

O’DEA, L., DWYER, N., CUMMINS, V. \& WRIGHT, D. 2011 Potentials and limitations of coastal web atlases. J. Coastal Conserv. 15(4):607-627. http://dx.doi.org/10.1007/s11852-011-0150-7

PHILliPS, A. \& WILLIAMSON, I. 1999. Spatial Data Infrastructure concepts. Aust. Surv. 44(1):20-28.

PROCTOR, R. \& HOWARTH, M.J. 2008. Coastal Observatories and operational oceanography: a European perspective. Mar. Technol. Soc. J. 42(3):10-13. http://dx.doi.org/10.4031/002533208786842534

RODRIGUES, M., FURTADO, V.V., TESSLER, M.G. \& MAHIQUES, M.M. (orgs.). 2001. Atlas Sedimentológico da plataforma continental do estado de São Paulo. Ed.Grupo de Oceanografia Geológica, IO-USP, 34 p.

SOBERÓN, J. \& PETERSON, A.T., 2004. Biodiversity informatics: managing and applying primary biodiversity data. Philos. Trans. Royal Soc. Lond. B. 359:689-698. http://dx.doi.org/10.1098/rstb.2003.1439

STRAIN, L., RAJABIFARD, A. \& WILLIAMSON, I.P. 2006. Spatial Data Infrastructure and Marine Administration. J. Mar. Policy 30(4):431-444. http://dx.doi.org/10.1016/j.marpol.2005.03.005

WRIGHT, D.J. 2009. Spatial data infrastructures for coastal environments in Remote Sensing and Geospatial Technologies for Coastal Ecosystem Assessment and Management. In Lecture Notes in Geoinformation and cartography (X. Yang, ed.). Springer, Berlin, p.91-112.

Recebido em 06/12/2012

Versão reformulada recebida em 10/05/2013

Publicado em 24/05/2013 